\title{
MODIFIED COORDINATE METHOD TO SOLVE MULTICRITERIA OPTIMIZATION PROBLEMS ON COMBINATORIAL CONFIGURATIONS
}

\author{
L. N. Koliechkina, ${ }^{a \dagger}$ O. A. Dvernaya, ${ }^{a \dagger}$ and A. N. Nagornaya ${ }^{b}$ \\ UDC 519.85
}

\begin{abstract}
We propose an approach to solve a multicriteria optimization problem on combinatorial configuration of permutations by using graph theory, taking into account the properties and structure of the set of permutations. The subprogram of the method of searching for configuration points that uses the coordinate method in the proposed modified approach is described.
\end{abstract}

Keywords: extremum problems, combinatorial configurations, extremum problems on combinatorial configurations, multicriteriality condition, coordinate method.

\section{INTRODUCTION}

Making optimal decisions is inevitable and necessary in various fields of human activity such as economics, production, designing, agriculture, etc. [1-4]. Depending on the complexity of problems and objectives, it can be interpreted as a solution of certain extremum problem of searching the maximum or minimum value of a given parameter to be optimized. Constructing the set of feasible solutions of the problem is characterized by the membership in a combinatorial set and influences the combinatorial properties of the problem considered on a certain combinatorial configuration [5]. Attaining the best result for several objectives simultaneously is determined by the multicriteriality condition and represents the problem as vector one, i.e., related to searching for the optimal values of the vector objective function by the choice from the set of feasible solutions.

As the number of optimization criteria increases, the problem complexity grows [6-11] and the need arises to develop a new approach to the solution of vector combinatorial problems since the general algorithm can not always be adapted and applied efficiently. Therefore, the form of its mathematical formulation and search for solution methods are important.

The present paper continues the studies in combinatorial optimization under multicriteriality condition [3, 8-12]. Our purpose is to develop a modified approach to the solution of the multicriteria problem on the set of permutations, to construct an algorithm for the modified approach, and to carry out a numerical experiment.

\section{FORMULATION OF THE EXTREMUM PROBLEM UNDER MULTICRITERIALITY CONDITION}

According to [7], an optimization problem can be generally represented as a tuple $\langle F, X, \Pi, D$, extr $\rangle$, where $F: X \rightarrow R^{1}$ is a given objective function, $R^{1}$ is a number axis, $X$ is decision space, $\Pi$ is a predicate that defines subset $D \subseteq X$ of feasible candidate solutions of the problem taking into account the conditions of its constraints and extr $\in\{\min , \max \}$ is the optimization direction. Then the problem can be formulated as follows: find $x^{*} \in D \subseteq X$ such that

$$
x^{*}=\arg \underset{x \in D \subseteq X}{\operatorname{extr}} F(x)
$$

${ }^{\mathrm{a} P o l t a v a}$ University of Economics and Trade, Poltava, Ukraine, ${ }^{\dagger} l u d a p l @ u k r . n e t ;{ }^{\dagger}$ rodionovaoa@mail.ru. ${ }^{\mathrm{b}}$ Ukrainian State University of Finance and International Trade, Kyiv, Ukraine,vpn2006@rambler.ru. Translated from Kibernetika i Sistemnyi Analiz, No. 4, July-August, 2014, pp. 154-161. Original article submitted October 9, 2013. 
This extremum problem is combinatorial if the decision space $X$ is defined as a certain combinatorial configuration. In the paper, as an example we consider the configuration of permutations.

The problem is multicriteria if the optimality criterion $F$ is vector, i.e., $F\left(f_{1}, f_{2}, . ., f_{n}\right)$. If these conditions are satisfied, problem (1) is an extremum combinatorial problem under multicriteriality condition. However, during the solution, an optimization problem with several criteria (a vector problem) can be reduced to a one-criterion problem by folding them. Therefore, the problem statement is to search for extremum values of the function on a certain combinatorial configuration under additional constraints. Finding the extremum value of the function in the absence of additional constraints is a quite easy problem since both the maximum and the minimum value can be found by arranging the coefficients in increasing order and determining the function value at the maximum or minimum point of the configuration [2].

We will consider a modified approach to the solution of the following problem: let a vector function $F\left(f_{1}, f_{2}, \ldots, f_{n}\right)$ be defined, where $f_{i}=\left\langle c_{i}, x_{i}\right\rangle \rightarrow$ extr, $i \in N_{n}$, on a combinatorial configuration $X=\{x\}$ and additional constraints $A_{i j} x_{j} \leq b_{j}$ be specified. It is necessary to find an element (or elements) of the configuration for which the function attains extremum (maximum, minimum) value and additional conditions of the problem (constraints) are satisfied.

The proposed approach differs from that described in [12]: first, the points of combinatorial configuration corresponding to the additional constraints are found, and the configuration element at which the function attains extremum value is determined among the set of these selected points [5]. And in the approach from [12], the solutions for which the function attains extremum are found first, conditions of the additional constraints are satisfied, and then the membership of the obtained solution in the considered combinatorial configuration is checked.

The coordinate method of localization of the value of linear function, modified to find the points satisfying additional constraints of the problem, allows reducing the number of points under consideration [6,7]. This approach is based on the properties of points that determine elements of the combinatorial configurations decomposed into subgraphs according to the selected type of nodes and represented as a subgraph scheme [7] where elements are ordered, and the value of the function on a certain subgraph is between the values at extreme nodes of the scheme. The graph is a network, where the upper left node is the source and the lower right node is the sink.

To use the coordinate method, it is necessary to determine the types of nodes for the graph deomposition. The decomposition is possible due to the hierarchy, i.e., a subgraph of a smaller dimension is a component of all the graphs of larger dimension. To determine the types of nodes, we will use a graph of dimension three. The number of its nodes is 3 !. We will decompose the graph into subgraphs with respect to these six types of nodes: (123), (132), (213), (231), (312), and (321). We will construct the subgraph mesh according to the following rules (on the example of the configuration of permutations of six elements):

- determine and fix the node type;

- fix the last sixth coordinate;

— in the upper row of the mesh, the next to last fifth coordinate "runs" all the possible values from the maximum to the minimum;

— in each column of the mesh, the fourth coordinate "runs" the remaining possible values from the maximum to the minimum;

- the first three coordinates are arranged in the order determined by the node type.

In solving the problem, such meshes are constructed for each node type. Their use is expedient since the values of the function with ordered coefficients at mesh nodes varies from greater to smaller one in the direction from left to right and from top downward. This allows rejecting a significant number of the configuration points at which it is impossible to attain the required values that satisfy the problem statement.

Using the graph theory to find solution methods for extremum problems on combinatorial configurations is a promising field of studies as it allows visualizing the set of solutions and, which is more important, using the properties of graphs of combinatorial configurations to structure and order not only points but also the values of functions and constraints.

\section{CONSTRUCTING THE ALGORITHM FOR THE MODIFIED COORDINATE SOLUTION METHOD}

This algorithm is intended to solve extremum problems on combinatorial sets under multicriteriality condition, for example, for the set of permutations. As we mentioned above, the main approach to the solution of multicriteria problems is 
to reduce the vector criterion to a scalar form. In the proposed algorithm, it implies selecting the weight factors of each optimality criterion using the formula

Thus, the problem becomes one-criterion.

$$
a_{i}^{k}=\frac{\sum_{s=1}^{m} \sigma_{i s}}{\sum_{r=1}^{m} \sum_{s=1}^{m} \sigma_{r s}}, i \in N_{n} .
$$

An advantage of the coordinate method is that it becomes unnecessary to find the function value by substituting the elements. This can be done by calculating the difference between the values at the previous and subsequent nodes, which reduces the number of necessary operations. Let us consider the algorithm.

\section{Algorithm of the modified coordinate solution method for extremum combinatorial problems under multicriteriality condition.}

1. Introduce coefficients of objective functions, additional constraints of the problem, and elements of the set on which the combinatorial configuration of permutations is constructed $\mathrm{d}$. 2. Transform the vector criterion into a linear function $f^{*}=\sum_{i=1} a_{i}^{k} f_{i} \rightarrow$ extr, where the weight factors of the new
optimality criterion are calculated by formula (2).

3. For each of the $k$ constraints find the points of the configuration of permutations corresponding to it, using the subprogram of the modified coordinate method.

Algorithm of the subprogram of the modified coordinate method.

Step 1. Set the initial values of the variables: $t=1, k=4$, and $i=6$.

Step 2. Fix the type of node $v_{t}=\left(i_{1}, i_{2}, i_{3}\right)$, where $i$ is subgraph number, $i_{1} \cup i_{2} \cup i_{3}=\{1,2,3\}$.

Step 3. Find the values as follows: $x_{s}=i ; x_{s-1}=\max \left\{N_{s} \backslash x_{s}\right\} ; x_{s-2}=\max \left\{N_{s} \backslash\left(x_{s}, x_{s-1}\right)\right\} ; \ldots ; x_{4}=\max \left\{N_{s} \backslash\left(x_{s}\right.\right.$, $\left.\left.x_{s-1}, \ldots, x_{5}\right)\right\}$. Arrange the numbers $\left\{N_{s} \backslash\left(x_{s}, x_{s-1}, \ldots, x_{4}\right)\right\}$ in increasing order $j_{1}<j_{2}<j_{3}$. Then $x_{1}=j_{i_{1}}, x_{2}=j_{i_{2}}$, and $x_{3}=j_{i_{3}}$ are the codes of the principal vertex, which we denote by $p_{1}$. The value of the constraint function in it for the given node type and fixed coordinate is maximum for the constructed network.

Step 4. Calculate the function value at the code of the principal node $f\left(p_{1}\right)$.

Step 5. Consider and arrange in decreasing order the values $x_{k}, k \in N_{k}: j_{k}>j_{k-1}>\ldots>j_{1}$. Decompose the graph in the direction of coordinate $x_{k}$ by carrying out the sequence of transpositions: $j_{k} \Leftrightarrow j_{k-1} \Leftrightarrow \ldots \Leftrightarrow j_{1}$, which lead to forming more $k-1$ codes of nodes $p_{2}, p_{3}, \ldots, p_{k}$, which are the codes of nodes of the upper line of the scheme.

Step 6. Find the function value on permutations by using their coordinates $f\left(p_{n}\right)=f\left(p_{n-1}\right)-\Delta_{n-1}$, $\Delta_{n-1}=\left(j_{n}-j_{n-1}\right)\left(c_{n}-c_{\mu(n-1)}\right)$, where $\mu(\lambda)$ is the number of the place of number $j_{\lambda}$ in the code of permutation $p_{n-1}$.

Step 7. Verify the following conditions:

- if $f\left(p_{m}\right) \geq y^{*}$ (the required values exist in the constructed subgraph), then include the $m$ th permutation in the further search and go to Step 8;

- if for all the found codes $f\left(p_{m}\right)<y^{*}$ and $i-1 \leq 1$ (the required values do not exist in the constructed subgraph, but not all the possible fixed coordinates for the selected node type are considered), then pass to subgraphs with the next fixed coordinate $x_{s}=i$ and then go to Step 2;

- if for all the found codes $f\left(p_{m}\right)<y^{*}$ and $i-1=0$ (the required values do not exist in the constructed subgraph and all the possible fixed coordinates for the selected node type are considered), then assign $i=6$, go to considering subgraphs with node type $v_{t+1}$ and to the next condition;

- if $t+1 \leq 6$ (not all the node types are considered), then go to Step 2; otherwise (for all the six node types subgraphs are already constructed) complete the algorithm for this constraint.

Step 8. Increase $k$ by one. If $k<s$, then go to Step 9; otherwise go to considering subgraphs with the next fixed coordinate $x_{s}=i$, i.e., to Step 2. (If $i-1=0$, then assign $i=6$ and go to considering subgraphs with node type $v_{t+1}$ provided that $t+1 \leq 6$; otherwise, complete the algorithm for this constraint.)

Step 9. Consider and arrange in decreasing order the values $x_{k}, k \in N_{k}: j_{k}>j_{k-1}>\ldots>j_{1}$. Decompose the graph along the coordinate $x_{k}$ by carrying out the sequence of transpositions $j_{k} \Leftrightarrow j_{k-1} \Leftrightarrow \ldots \Leftrightarrow j_{1}$, which lead to forming more $k-1$ codes of the nodes $q_{2}, q_{3}, \ldots, q_{k}$. 
Step 10. Find the function value on these permutations using their coordinates $f\left(q_{n}\right)=f\left(q_{n-1}\right)-\delta_{n-1}$, $\delta_{n-1}=\left(j_{n}-j_{n-1}\right)\left(c_{n}-c_{\mu(n-1)}\right)$, where $\mu(\lambda)$ is the number of the place of number $j_{\lambda}$ in the permutation code $q_{n-1}$.

Step 11. If $f\left(q_{n}\right)>y^{*} \forall n \in N_{k}$, then go to Step 8. If $f\left(q_{n}\right) \leq y^{*}$, then save the code of the node $q_{n}$.

4. Obtain $k$ sets $D_{i} \subset X$, where $i \in N_{k}$.

5. Find the intersection $D^{*}=D_{1} \cap D_{2} \cap \ldots \cap D_{k}$.

6. Calculate the function value at points $x \in D^{*}$ and compare them by choosing the appropriate extremum value (maximum or minimum).

7. Find the value of functions that compose the vector criterion. End the algorithm.

Let us consider the algorithm using the following example.

Example. Let it be necessary to solve the following problem: find points of the set of permutations of elements $A=(1,2,3,4,5,6)$, in which the maximum values of functions

$$
\begin{aligned}
& f_{1}(x)=x_{1}+4 x_{2}+15 x_{3}+3 x_{4}+15 x_{5}+3 x_{6}, \\
& f_{2}(x)=4 x_{1}+3 x_{2}+2 x_{3}+11 x_{4}+9 x_{5}+15 x_{6}, \\
& f_{3}(x)=x_{1}+8 x_{2}+7 x_{3}+16 x_{4}+18 x_{5}+27 x_{6}
\end{aligned}
$$

are attained under the constraints

$$
\left\{\begin{array}{l}
2 x_{1}+3 x_{2}+4 x_{3}+5 x_{4}+6 x_{5}+8 x_{6} \leq 113 \\
3 x_{1}+x_{2}+5 x_{3}+2 x_{4}+3 x_{5}+7 x_{6} \leq 91
\end{array}\right.
$$

Solution. Let us transform the vector criterion of the problem $F\left(f_{1}, f_{2}, f_{3}\right)$ into a scalar one. To this end, we determine the weight factors of each function provided that they are equally important: $\alpha_{1}=\alpha_{2}=\alpha_{3}=1 / 3$. Then the objective function is the following:

$$
f^{*}(x)=2 x_{1}+5 x_{2}+8 x_{3}+10 x_{4}+14 x_{5}+15 x_{6} .
$$

Let us pass to the first constraint. Choose sequentially the node types (123), (132), (213), (231), (312), and (321) and fix the last coordinate for each type.

For the constraint $2 x_{1}+3 x_{2}+4 x_{3}+5 x_{4}+6 x_{5}+8 x_{6} \leq 113$ (function $g_{1}(x)=2 x_{1}+3 x_{2}+4 x_{3}+5 x_{4}+6 x_{5}+8 x_{6}$ ), determine the first type of node (123) and fix the last coordinate $x_{6}=6$. Find the function value at the upper left node by substituting its coordinates into the function $g_{1}(1,2,3,4,5,6)=118$. This means that the values of the constraint function at all the other elements of this scheme are less than 118, i.e., it can contain points that satisfy the constraints. We obtain the passage from the upper left to the upper right node by successive permutations of elements $a_{5} \Leftrightarrow a_{4}, a_{5} \Leftrightarrow a_{3}, a_{5} \Leftrightarrow a_{2}$, $a_{5} \Leftrightarrow a_{1}$. Then the values of differences of nodes according to the coordinate method are calculated as follows: $\Delta_{1}=(5-4) *(6-5)=1, \Delta_{2}=(4-3) *(6-4)=2, \Delta_{3}=(3-2) *(6-3)=3$, and $\Delta_{4}=(2-1) *(6-2)=4$. The function values are, respectively, as follows:

$$
\begin{gathered}
g_{1}\left(p_{1}\right)=g_{1}(1,2,3,4,5,6)=118, \\
g_{1}\left(p_{2}\right)=g_{1}(1,2,3,5,4,6)=118-\Delta_{1}=118-1=117, \\
g_{1}\left(p_{3}\right)=g_{1}(1,2,4,5,3,6)=117-\Delta_{2}=117-2=115, \\
g_{1}\left(p_{4}\right)=g_{1}(1,3,4,5,2,6)=115-\Delta_{3}=115-3=112, \\
g_{1}\left(p_{5}\right)=g_{1}(2,3,4,5,1,6)=112-\Delta_{4}=112-4=108 .
\end{gathered}
$$

Figure 1 shows the scheme with the found values of the constraint function (shaded areas) and with points that satisfy the first constraint (unshaded areas). It is obvious that columns 4 and 5 of the scheme (Fig. 1) contain the points that satisfy this constraint, as their maximum values (112 and 108) are less than the given number 113. Columns 1, 2, and 3 may contain such points. We obtain the function values from the upper left to the lower left node by successive permutations of elements $a_{4} \Leftrightarrow a_{3} \Leftrightarrow a_{2} \Leftrightarrow a_{1}$. The function values at these points become as follows: $118 \rightarrow 117 \rightarrow 115 \rightarrow 112$. Hence, the points of the last line of the scheme completely satisfy the first constraint. Lines 2 and 3 should be considered additionally, having found the function values at the nearest points. When finding the value equal to or smaller than 113, the analysis can be stopped since the result will be unambiguous. 


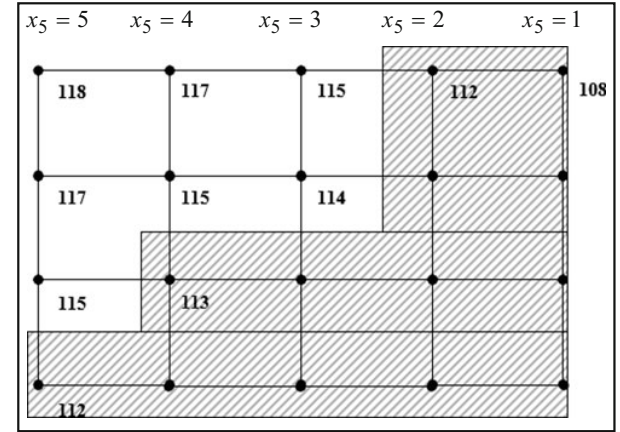

Fig. 1. Scheme for the first constraint for the node type (123) and $x_{6}=6$.

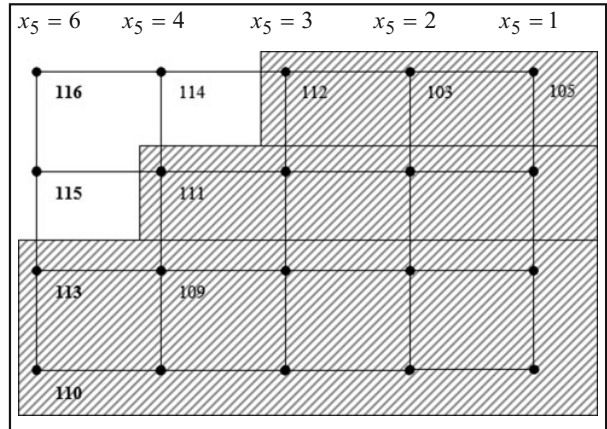

Fig. 2. Scheme for the first constraint for the node type (123) and $x_{6}=5$.
TABLE 1. The Value of the First Constraint for Node Type (123)

\begin{tabular}{|c|c|c|}
\hline $\begin{array}{c}\text { Last } \\
\text { coordinate }\end{array}$ & $\begin{array}{c}\text { Point } \\
\text { coordinates }\end{array}$ & $\begin{array}{c}\text { Function } \\
\text { value }\end{array}$ \\
\hline 4 & $(123564)$ & 113 \\
3 & $(124563)$ & 109 \\
2 & $(134562)$ & 104 \\
1 & $(234561)$ & 98 \\
\hline
\end{tabular}

TABLE 2. The Value of the First Constraint at the upper Left Points of the Schemes

\begin{tabular}{|c|c|c|c||c|c|c|c|}
\hline Node type & $\begin{array}{c}\text { The last } \\
\text { coordinate }\end{array}$ & $\begin{array}{c}\text { Point } \\
\text { coordinates }\end{array}$ & $\begin{array}{c}\text { Function } \\
\text { value }\end{array}$ & Node type & $\begin{array}{c}\text { The last } \\
\text { coordinate }\end{array}$ & $\begin{array}{c}\text { Point } \\
\text { coordinates }\end{array}$ & $\begin{array}{c}\text { Function } \\
\text { value }\end{array}$ \\
\hline$(132)$ & 4 & $(132564)$ & 112 & $(213)$ & 2 & $(314562)$ & 102 \\
$(132)$ & 3 & $(142563)$ & 107 & $(213)$ & 1 & $(324561)$ & 97 \\
$(132)$ & 2 & $(143562)$ & 103 & $(312)$ & 4 & $(312564)$ & 110 \\
$(132)$ & 1 & $(243561)$ & 97 & $(312)$ & 3 & $(412563)$ & 104 \\
$(231)$ & 4 & $(231564)$ & 110 & $(312)$ & 2 & $(413562)$ & 100 \\
$(231)$ & 3 & $(241563)$ & 105 & $(312)$ & 1 & $(423561)$ & 95 \\
$(231)$ & 2 & $(341562)$ & 99 & $(321)$ & 4 & $(321564)$ & 109 \\
$(231)$ & 1 & $(342561)$ & 95 & $(321)$ & 3 & $(421563)$ & 103 \\
$(213)$ & 4 & $(213564)$ & 112 & $(321)$ & 2 & $(431562)$ & 98 \\
$(213)$ & 3 & $(214563)$ & 108 & $(321)$ & 1 & $(432561)$ & 94 \\
\hline
\end{tabular}

Next, we will consider the schemes for the established node type (123) by sequentially fixing the last coordinate $x_{6}=5, x_{6}=4, x_{6}=3, x_{6}=2$, and $x_{6}=1$. The results of calculations for $x_{6}=5$ are represented by Fig. 2. For the other coordinates, we obtain the values at the upper left point of the scheme.

It follows from Table 1 that the maximum function value of the schemes does not exceed the given number 113, i.e., all the points of the scheme satisfy the first constraint.

The general result for similar studies for the other node types is presented in Table 2.

Let us consider the second constraint $3 x_{1}+x_{2}+5 x_{3}+2 x_{4}+3 x_{5}+7 x_{6} \leq 91$. Find the function with the coordinates arranged in increasing order $g_{2}(x)=x_{1}+2 x_{2}+3 x_{3}+3 x_{4}+5 x_{5}+7 x_{6}$. Determine the node type (123) and fix the coordinate $x_{6}=6$. The results are presented as a scheme in Fig. 3 and in Table 3.

Let us determine the node type (132) and fix the coordinate $x_{6}=6$. The results of the analysis are presented as a scheme in Fig. 4 and in Table 3.

From the latter we can see that the other schemes satisfy the second constraint at all the points.

Analyzing the results of calculations for the second constraint, we conclude that only four points do not satisfy this constraint: (241536), (245136), (215436), and (214536). Thus, for the intersection of the sets of feasible values, according to the first and second constraints, it will suffice to delete these points on the schemes for the first constraint.

Let us find the maximum points of each scheme and calculate the value of the objective function at these points, wherefrom we can determine the optimal solution. Thus, the maximum value of the objective function is attained at the point (135246) and is 234 (Table 4). 


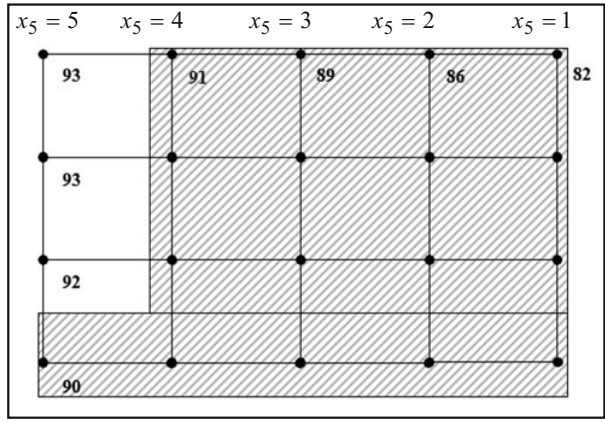

Fig. 3. Scheme for the second constraint for the node type (123) for $x_{6}=6$.

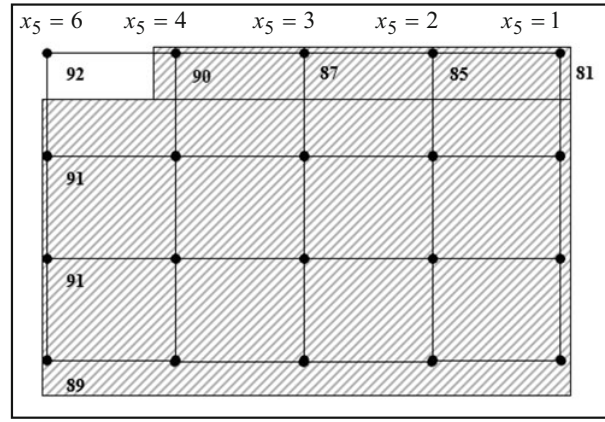

Fig. 4. Scheme for the second constraint for the node type (132)

$$
\text { for } x_{6}=6 \text {. }
$$

TABLE 3. The Value of the Second Constraint at the upper Left Points of the Schemes

\begin{tabular}{|c|c|c|c||c|c|c|c|}
\hline Node type & $\begin{array}{c}\text { The last } \\
\text { coordinate }\end{array}$ & $\begin{array}{c}\text { Point } \\
\text { coordinates }\end{array}$ & $\begin{array}{c}\text { Function } \\
\text { value }\end{array}$ & Node type & $\begin{array}{c}\text { The last } \\
\text { coordinatea }\end{array}$ & $\begin{array}{c}\text { Point } \\
\text { coordinates }\end{array}$ & $\begin{array}{c}\text { Function } \\
\text { value }\end{array}$ \\
\hline$(123)$ & 5 & $(123465)$ & 91 & $(213)$ & 5 & $(213465)$ & 90 \\
$(123)$ & 4 & $(123564)$ & 87 & $(213)$ & 4 & $(213564)$ & 86 \\
$(123)$ & 3 & $(124563)$ & 83 & $(213)$ & 3 & $(214563)$ & 82 \\
$(123)$ & 2 & $(134562)$ & 78 & $(213)$ & 2 & $(314562)$ & 76 \\
$(123)$ & 1 & $(234561)$ & 72 & $(213)$ & 1 & $(324561)$ & 71 \\
$(132)$ & 5 & $(132465)$ & 90 & $(312)$ & 6 & $(312456)$ & 90 \\
$(132)$ & 4 & $(132564)$ & 86 & $(312)$ & 5 & $(312465)$ & 88 \\
$(132)$ & 3 & $(142563)$ & 81 & $(312)$ & 4 & $(312564)$ & 84 \\
$(132)$ & 2 & $(143562)$ & 77 & $(312)$ & 3 & $(412563)$ & 78 \\
$(132)$ & 1 & $(243561)$ & 71 & $(312)$ & 2 & $(413562)$ & 74 \\
$(231)$ & 6 & $(231456)$ & 90 & $(312)$ & 1 & $(423561)$ & 69 \\
$(231)$ & 5 & $(231465)$ & 88 & $(321)$ & 6 & $(321456)$ & 89 \\
$(231)$ & 4 & $(231564)$ & 84 & $(321)$ & 5 & $(321465)$ & 87 \\
$(231)$ & 3 & $(241563)$ & 79 & $(321)$ & 4 & $(321564)$ & 83 \\
$(231)$ & 2 & $(341562)$ & 73 & $(321)$ & 3 & $(421563)$ & 77 \\
$(231)$ & 1 & $(342561)$ & 69 & $(321)$ & 2 & $(431562)$ & 72 \\
$(213)$ & 6 & $(213456)$ & 92 & $(321)$ & 1 & $(432561)$ & 68 \\
\hline
\end{tabular}

TABLE 4. The Values of the Objective Functions

\begin{tabular}{|c|c||c|c||c|c|}
\hline $\begin{array}{c}\text { Point } \\
\text { coordinates }\end{array}$ & Function value & $\begin{array}{c}\text { Point } \\
\text { coordinates }\end{array}$ & Function value & $\begin{array}{c}\text { Point } \\
\text { coordinates }\end{array}$ & Function value \\
\hline$(134526)$ & 217 & $(134265)$ & 228 & $(132465)$ & 232 \\
$(135246)$ & 234 & $(123564)$ & 230 & $(132564)$ & 227 \\
$(234156)$ & 221 & $(142536)$ & 220 & $(142563)$ & 217 \\
$(124635)$ & 221 & $(152346)$ & 219 & $(231536)$ & 209 \\
$(126345)$ & 221 & $(243156)$ & 218 & $(241356)$ & 222 \\
\hline
\end{tabular}

\section{CONCLUSIONS}

We have analyzed complex vector problems on the combinatorial configuration of permutations. We have proposed a modified approach to the solution of problems based on the coordinate method, have formulated a solution algorithm for the vector function on the combinatorial configuration of permutations, and have carried out numerical experiments. The modified approach has a practical value and is of interest in the construction and development of the structured method to solve problems on various combinatorial configurations under additional complex constraints. 


\section{REFERENCES}

1. V. I. Baranov and B. S. Stechkin, Extremum Combinatorial Problems and their Applications [in Russian], Fizmatlit, Moscow (2004).

2. K. A. Rybnikov, An Introduction to Combinatorial Analysis [in Russian], Izd. Moskov. Univ., Moscow (1985).

3. N. V. Semenova, L. N. Kolechkina, and A. N. Nagirna, "An approach to solving discrete vector optimization problems over a combinatorial set of permutations," Cybern. Syst. Analysis, 44, No. 3, 441-451 (2008).

4. I. V. Sergienko and M. F. Kaspshitskaya, Models and Methods for Computer Solution of Combinatorial Optimization Problems [in Russian], Naukova Dumka, Kyiv (1981).

5. V. N. Sachkov, Combinatorial Methods of Discrete Mathematics [in Russian], Nauka, Moscow (1977).

6. G. A. Donets and L. N. Kolechkina, "Method of ordering the values of a linear function on a set of permutations," Cybern. Syst. Analysis, 45, No. 2, 204-213 (2009).

7. G. P. Donets and L. M. Kolechkina, Extremum Problems on Combinatorial Configurations [in Ukrainian], PUET, Poltava (2011).

8. G. A. Donets and L. N. Kolechkina, "On one problem of optimization of a linear fractional function on permutations," J. Autom. Inform. Sci., 42, No. 4, 1-12 (2010).

9. N. V. Semenova, L. N. Kolechkina, and A. N. Nagirna, "Vector optimization problems with linear criteria over a fuzzy combinatorial set of alternatives," Cybern. Syst. Analysis, 47, No. 2, 250-259 (2011).

10. N. V. Semenova, L. N. Kolechkina, and A. N. Nagornaya, "Solving multicriteria combinatorial optimization problems on the set of polypermutations," Dop. NANU, No. 6, 41-48 (2010).

11. L. N. Kolechkina, "Justifying the structured method of localizing the value of a linear function specified on a combinatorial configuration of permutations," Dinamich. Sistemy, No. 27, 67-80 (2009).

12. L. N. Kolechkina and E. A. Rodionova, "Multicriteria combinatorial optimization problems on a set of polypermutations," Cybern. Syst. Analysis, 44, No. 2, 276-282 (2008). 